\title{
Importanza dei flussi informativi nella sorveglianza delle malattie infettive soggette a notifica
}

\section{Angelo Sala', Cristina Pianetta', Donatella Polvara', Roberto Vaiani', Folsi Maria Fiorenza², Cristina Tentori' ${ }^{2}$, Laura Chiappa ${ }^{2}$}

\author{
' Laboratorio Patologia Clinica II - Microbiologia, Azienda Ospedaliera “A. Manzoni”, Lecco. \\ ${ }^{2}$ Direzione Sanitaria, Azienda Ospedaliera “A. Manzoni”, Lecco.
}

\section{Importance of active communication in notifiable infectious diseases surveillance}

Key words: Infections diseases surveillance, Hospital Office Control, Medical Direction

\section{SUMMARY}

A system of epidemiological surveillance of infectious diseases is performed according the actual law and notified to Regional Register after the isolation done in the Microbiology lab. The system is operating in our hospital. The notification is done after 12-48 hours in the module ISO 9000 to the Sanitary Surveillance Office and the Office Control Hospital Infection and regards name of patients, provenience, collection time, time of diagnostic performance, and diagnostic bacteriological evaluation. All data regarding the control procedures are included in the same protocol.

The microbiology data is sent immediately by phone to the medical staff who is involved to the compilation of specific protocol (MB form) sent directly from medical staff to the Medical Direction.

The Medical Direction is involved to advise the Local Sanitary Authority (Azienda Sanitaria Locale, ASL) in all cases in which is necessary to send to reference laboratories some material from patients (smears, coltures, etc) and to inform National Authority.

From January 2002 to April 2003364 cases were involved and the present study is involved in the analysis of the system. From 62 coltural examination 43 involved Salmonella spp and from 39 serological data 19 involved EB disease. Clostridium difficile was detected in 90 cases (toxins A and B) and Rotavirus in 36 cases. Mycobacteria were detected 22 cases and Parasitic disease in 14 cases.

The system is highly recomanded surveillance of notifiable infectious diseases in hospital and for control of hospital infections. The absence of notification is not allowed.

\section{INTRODUZIONE}

Tra gli indicatori di qualità di ogni struttura ospedaliera accreditata deve figurare la pianificazione di una attività di prevenzione e controllo delle infezioni nosocomiali $(4,8,19)$.

In particolare, per sorvegliare, trattare e monitorare le infezioni epidemiologicamente più rilevanti, occorre avvalersi di un sistema informativo locale $(11,12)$.

Detto sistema consiste nello sviluppo di una rete di comunicazione in grado di rispondere alle esigenze di tipo documentale di qualsivoglia evento epidemiologico: sentinella, epidemico o di malattia infettiva soggetta a denuncia $(1,5,7)$.

La sorveglianza basata sul dato del Laboratorio di Microbiologia consente di definire il quadro epidemiologico locale nel suo andamento temporale, purché possegga i requisiti informatici per la elaborazione delle informazioni di cui deve essere la fonte principale $(10,18)$.

Lo "starter" d'avvio della sorveglianza è la raccolta di dati clinico - microbiologici utili e affidabili (9).
Questi ultimi devono essere trasferiti- secondo modalità convenzionale od elettronica - agli utilizzatori finali di competenza in relazione al tipo di segnalazione $(13,15)$.

La definizione dei flussi informativi, la tracciabilità dei percorsi di comunicazione, l'individuazione del nucleo operativo e il richiamo alle responsabilità specifiche costituiscono il fulcro attorno a cui ruota il modello organizzativo della circolazione dei reports a partire da un livello locale per poi raggiungere quelle Istituzioni di Sanità Pubblica a livello territoriale e centrale $(2,3,6)$.

Dal 2001 è stato avviato presso la nostra Azienda un sistema di sorveglianza epidemiologica delle malattie infettive soggette a notifica obbligatoria in accordo alle normative vigenti (D.M. del 15/12/1990 e del 18/06/1996 - Circolare Regione Lombardia ${ }^{\circ} 15 / \mathrm{SAN}$ del18/06/1996).

Detto sistema si sviluppa a partire dalla segnalazione tempestiva - tra le 12 e le $48 \mathrm{~h}$ - del microrganismo ritenuto responsabile della patologia infettivologica riferita alle cinque classi dell'elenco ufficiale in grado di costituire pericolo per la 
Sanità Pubblica.

La segnalazione per via breve viene effettuata con modalità di comunicazione cartacea all'Ufficio Sorveglianza Sanitaria e Controllo Infezioni Ospedaliere (USS-CIO) della Direzione Sanitaria (D.S.) su apposito modulo normato ISO 9000.

E allo studio l'opportunità d'uso di un supporto informatico che consenta l'inoltro dei dati in tempo reale e la loro archiviabilità.

Il modulo reca le informazioni anagrafiche del paziente, la tipologia, la provenienza e la data di invio del campione, la data di esecuzione dell'analisi e il criterio diagnostico con cui è stata condotta l'indagine.

Tale criterio si riferisce alle cinque categorie in cui si compendia l'accertamento diagnostico del Laboratorio, ossia l'Esame Colturale, l'Esame Sierologico, la ricerca di Antigeni/Tossine, la ricerca dei Micobatteri e l'Esame Parassitologico.

\section{FLUSSI INFORMATIVI E METODI}

Per valutare la funzionalità del nostro modello organizzativo che poggia sull'alleanza tra le diverse competenze espresse sul campo, sono state analizzate le segnalazioni per via breve effettuate dalla Microbiologia nel periodo Gennaio 2002 - Aprile 2003 in base agli accertamenti diagnostici riferiti ai pazienti in regime di ricovero ordinario e in regime di day-hospital.

Sono stati esclusi dalla seguente analisi retrospettiva la casistica ambulatoriale, i cluster epidemici, i patogeni sentinella segnalati in modo differenziato in quanto eventi ascrivibili ad un sistema di allerta per la sorveglianza delle infezioni ospedaliere (14).

Il primo step informativo è a carico del Laboratorio di Microbiologia che procede all'inoltro della scheda di segnalazione (figura I).

Il successivo percorso si materializza nella compilazione di una scheda di rilevazione dei dati del paziente, da parte dell'USS-CIO, corredata da: informazioni cliniche raccolte direttamente dalla U.O. interessata, altre informazioni atte a stabilire se trattasi di infezione comunitaria od ospedaliera, provvedimenti disposti, precauzioni adottate in caso di contatto e strategie conseguenti di profilassi specifiche (figura II).

L'infermiera epidemiologa individua le aree prioritarie d'intervento e controllo in merito alla malattia infettiva osservata, mobilitando le risorse disponibili nell'applicazione di protocolli già precodificati e misure di precauzione universale per l'allontanamento del rischio occupazionale.

Il dato microbiologico tempestivamente comunicato per via telefonica alla U.O. interessata, attiva il medico curante che procede alla notifica di malattia infettiva alla D.S. tramite il tradizionale modello MB.

La D.S. a sua volta inoltra il dato all'ASL locale e/o ad altre Autorità Sanitarie (Assessorato alla Sanità - Regione Lombardia, Istituto Superiore di Sanità, Ministero della Salute, Istituto di Virologia) nel caso di sindromi infettive per le quali necessita la compilazione di apposite schede di rilevazione e/o dell'invio di ceppi batterici, vetrini o campioni biologici in caso di sospetto/accertamento di meningite meningococcica, leptospirosi, listeriosi, legionellosi, tubercolosi, lebbra, malaria, AIDS, malattia da prioni e da ultimo la SARS.

\section{RISULTATI}

È stata condotta l'analisi stratificata per U.O., materiale clinico, tipo di indagine diagnostica sulle 364 segnalazioni per via breve effettuate dal Laboratorio di Microbiologia nel periodo osservato.

I microrganismi più frequentemente isolati dai 62 esami colturali sono riferibili: 43 a Salmonella spp di cui 25 ceppi di gr.B, 8 a Campylobacter spp, 2 a Neisseria meningitidis, 4 a Listeria monocytogenes e 5 a Streptococcus pyogenes, ripartiti per U.O. e materiale come in tabella 1.

Per i Reparti Intensivi la distribuzione dei microrganismi isolati è risultata essere: 1 ceppo di Streptococcus pyogenes, 1 ceppo di Listeria monocytogenes per la Patologia Neonatale, 1 ceppo di Streptococcus pyogenes e 1 ceppo di Salmonella gr.B per la Rianimazione mentre in Neurorianimazione vi è stato l'isolamento di 1 ceppo di Neisseria meningitidis.

Non sono stati segnalati i casi di positività al Tampone Faringeo per Streptococcus pyogenes responsabile di faringotonsillite.

Le 39 infezioni soggette a notifica accertate tramite esame sierologico con sieroconversione sono distribuite per U.O. e tipo di infezione in tabella 2.

Le infezioni soggette a notifica accertate tramite ricerca di antigeni/tossine sono risultate rispettivamente 81 e 90 secondo la distribuzione indicata in tabella 3.

In tabella 4 sono riportati i 22 casi di TBC e Micobatteriosi stratificati per reparto e diagnosticati sulla base dell'indagine impiegata in primis tra quelli saggiati: es. microscopico, es. colturale, sonda genica..

In tabella 5 sono descritti i casi di parassitosi intestinali ed ematiche rispettivamente assommanti a 9 e a 5 secondo una stratificazione per U.O. Per le parassitosi ematiche la coppia emoscopia - ICT (es. immunocromatografico - BINAX) è conteggiato come unico esame. 


\begin{tabular}{|c|c|c|c|c|c|}
\hline \multicolumn{2}{|c|}{$\begin{array}{l}\text { Ospedale di Lecco } \\
\text { Azienda Ospedaliera } \\
\text { Provinciale del } \\
\text { S.S.N. } \\
\end{array}$} & \multicolumn{3}{|c|}{$\begin{array}{c}\text { SCHEDA DI SEGNALAZIONE } \\
\text { DEI MICROORGANISMI IMPLICATI IN PATOLOGIE } \\
\text { INFETTIVE SOGGETTE A NOTIFICA }\end{array}$} & \multirow[t]{2}{*}{ DL 27.40} \\
\hline \multicolumn{5}{|c|}{ UNITA' OPERATIVA DI MICROBIOLOGIA } & \\
\hline \multirow[t]{4}{*}{ PAZIENTE/I } & 1. & & REPARTO: & 1. & \\
\hline & 2. & & & 2. & \\
\hline & 3. & & & 3. & \\
\hline & 4. & & & 4. & \\
\hline
\end{tabular}

\begin{tabular}{|c|c|c|c|c|c|c|c|c|c|c|}
\hline CAMPIONE/I & 1. $\square$ & $2 . \square$ & 3. $\square$ & 4. $\square$ & FECI & 1. $\square$ & 2. $\square$ & 3. $\square$ & 4. $\square$ & SANGUE \\
\hline & $1 . \square$ & 2. $\square$ & 3. $\square$ & 4. $\square$ & URINE & 1. $\square$ & 2. $\square$ & $3 . \square$ & 4. $\square$ & LIQUOR \\
\hline & $1 . \square$ & 2. $\square$ & 3. $\square$ & 4. $\square$ & T. FARINGEO & $1 . \square$ & $2 . \square$ & 3. $\square$ & 4. $\square$ & SIERO \\
\hline & $1 . \square$ & 2. $\square$ & 3. $\square$ & 4. $\square$ & MAT. BIOPTICO & $1 . \square$ & $2 . \square$ & 3. $\square$ & 4. $\square$ & ESPETTORATO \\
\hline & 1. $\square$ & 2. $\square$ & 3. $\square$ & 4. $\square$ & 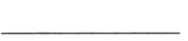 & & & & & \\
\hline
\end{tabular}

\begin{tabular}{|l|l|l|l|l|}
\hline $\begin{array}{l}\text { DATA } \\
\text { PRELIEVO }\end{array}$ & 1. & 2. & 3. & 4. \\
\hline
\end{tabular}

\begin{tabular}{|c|c|c|c|c|c|c|c|c|c|c|c|c|}
\hline ESAME & 1. $\square$ & 2. $\square$ & 3. $\square$ & 4. $\square$ & \multicolumn{3}{|c|}{ COLTURALE } & 1. $\square$ & 2. $\square$ & 3. $\square$ & 4. $\square$ & PARASSITOLOGICO \\
\hline & 1. $\square$ & 2. $\square$ & 3. $\square$ & 4. $\square$ & \multicolumn{3}{|c|}{ MICROSCOPICO } & 1. $\square$ & 2. $\square$ & 3. $\square$ & 4. $\square$ & PLASMODI MALARIA \\
\hline & 1. $\square$ & 2. $\square$ & 3. $\square$ & 4. $\square$ & \multicolumn{3}{|c|}{ SIEROLOGICO } & 1. $\square$ & 2. $\square$ & 3. $\square$ & \multicolumn{2}{|c|}{$\begin{array}{l}\text { 4. } \square \text { TOSSINE A,B } \\
\text { CLOSTRIDIUM DIFFICILE }\end{array}$} \\
\hline & $\begin{array}{l}\text { RICE } \\
\text { 1. } \square \\
1 . \square\end{array}$ & $\begin{array}{l}\text { A ANTI } \\
2 \square \\
2 . \square\end{array}$ & $\begin{array}{l}\text { NI CA } \\
\text { 3. } \square \\
\text { 3. } \square\end{array}$ & $\begin{array}{r}\text { LARI } \\
4 . \square \\
4 . \square\end{array}$ & URI & & & $\begin{array}{l}\text { RICF } \\
1 . \square \\
1 . \square \\
1 . \square\end{array}$ & $\begin{array}{l}\mathrm{A} \mathbf{A N} \\
2 . \square \\
2 . \square \\
2 . \square\end{array}$ & $\begin{array}{c}\text { NE PER } \\
\text { 3. } \square \\
\text { 3. } \square \\
\text { 3. } \square\end{array}$ & $\begin{array}{l}4 . \square \\
4 . \square \\
4 . \square\end{array}$ & $\begin{array}{l}\text { RSV } \\
\text { ROTAVIRUS } \\
\text { ADENOVIRUS }\end{array}$ \\
\hline & \multicolumn{12}{|c|}{ BAAR / MICOBATTERI } \\
\hline & \multicolumn{4}{|c|}{ ES. MICROSCOPICO } & 1. $\square$ & $2 . \square$ & 3. $\square$ & \multicolumn{2}{|c|}{ 4. $\square$} & & & \\
\hline & \multicolumn{3}{|c|}{ ES. COLTURALE } & & 1. $\square$ & 2. $\square$ & 3. $\square$ & \multicolumn{2}{|c|}{ 4. $\square$} & & & \\
\hline & \multicolumn{3}{|c|}{ SONDA GENICA } & & 1. $\square$ & 2. $\square$ & 3. $\square$ & \multicolumn{2}{|c|}{ 4. $\square$} & & & \\
\hline
\end{tabular}

\begin{tabular}{|l|l|l|}
\hline $\begin{array}{l}\text { MICROORGANISMO/PARASSITA/ } \\
\text { ANTIGENE/TITOLO } \\
\text { ANTICORPALE ACCERTATO/I: }\end{array}$ & 1. & 3. \\
\hline & 2. & 4. \\
\hline
\end{tabular}

DATA REFERTO:

FIRMA:

Fig. I 


$$
\begin{aligned}
& \text { OSPEDALE ALESSANDRO } \\
& \text { MANZONI -LECCO } \\
& \text { PRESIDIO DELL'AZIENDA } \\
& \text { OSPEDALIERA } \\
& \text { "OSPEDALE DI LECCO" } \\
& \text { U.O. Direzione Sanitaria di Presidio }
\end{aligned}
$$

\section{SCHEDA DI RILEVAZIONE DATI DEI PAZIENTI CON MALATTIE INFETTIVE SOGGETTE A NOTIFICA E INFEZIONI OSPEDALIERE}

\section{Ufficio Sorveglianza Sanitaria e Controllo Infezioni Ospedaliere}

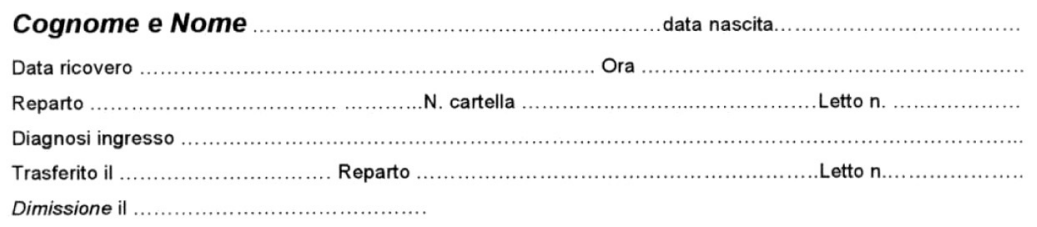

INFEZIONE RISCONTRATA:

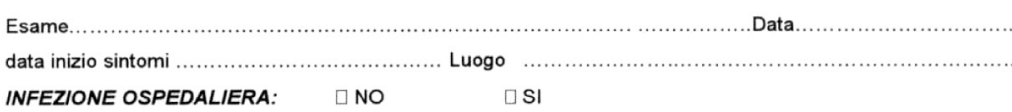

\section{SEGNALAZIONE LABORATORIO DI MICROBIOLOGIA:}

$\square$ pervenuta il NOTIFICA M. INFETTIVA:

$\square$ pervenuta il

$\square$ sollecitata il

\section{PROCEDURE INVASIVE:}

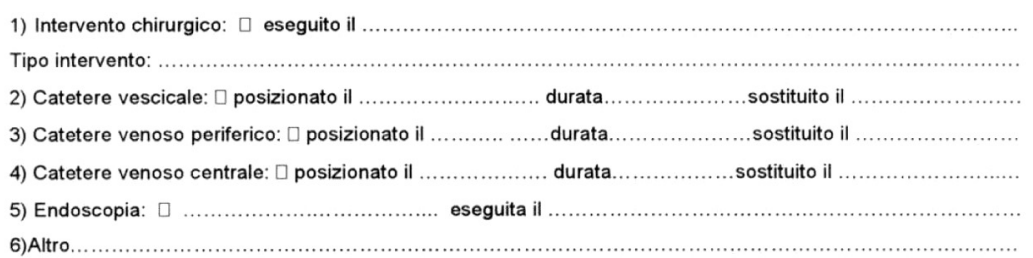

\section{PROVVEDIMENTI PRECAUZIONALI ADOTTATI:}

$\square$ No $\quad \square \mathrm{Si}$ (specificare quali)

Tipo isolamento

Dispositivi di Protezione Individuale

Altro

CONTATTI INTERPERSONALI: $\square$ NO $\square$ SI

\begin{tabular}{|l|l|}
\hline Nominativi & Provvedimenti disposti per contatti: \\
\hline 1. & \\
\hline 2. & \\
\hline
\end{tabular}

\section{CONDIZIONI GENERALI DEL PAZIENTE:}

Paziente autosufficiente $\square \quad$ Paziente non autosufficiente $\square$

Paziente parzialmente autosufficiente $\square$ Uso del bagno $\square$ Uso del bagno assistito $\square$

ANTIBIOTICOTERAPIA:

DIETA:

ALTRE NOTIZIE:

Data

Firma:

Fig. 2 
Tabella I. Microrganismi implicati in patologie infettive soggette a notifica isolati dalle colture di materiali clinici

\section{REPARTI/MATERIALI}

\begin{tabular}{llll}
\cline { 2 - 3 } MICRORGANISMI & PEDIATRIA NEFRO CARDIO T.INTENSIVE & UROLOGIA CH M.INF. MEDICINE OST.
\end{tabular}
gruppi

F S

A
B
C
D
E

$\begin{array}{llll}1 & & & \\ 11 & & 2 & 1 \\ 3 & 2 & & \\ 6 & & \end{array}$

Campylobacter spp.

Neisseria. $m$

Listeria $m$.

Streptococcus $p$.

2

MATERIALI: F=FECI; S=SANGUE; ESP=ESPETTORATO; LQ=LIQUOR;T.O.=TAMPONE OMBELICALE; EP=ESSUDATO PURULENTO; REPARTI:TERAPIE INTENSIVE = PATOLOGIA NEONATALE, RIANIMAZIONE, NEURORIANIMAZIONE NEFRO.= NEFROLOGIA; CARDIO.= CARDIOLOGIA; CH.= CHIRURGIE; M.INF =MALATTIE INFETTIVE; OST.= OSTETRICIA

Tabella 2. Malattie infettive soggette a notifica accertate con esame sierologico

INFEZIONI

MONONUCLEOSI MYCOPLASMA P. ROSOLIA

SIFILIDE INFEZIONE DA CMV

TOXOPLASMOSI

LEPTOSPIROSI

TOTALE

\begin{tabular}{|c|c|c|c|c|c|}
\hline MEDICINE & DER MATOLOGIA & MAL. INFETTIVE & PEDIATRIA & OTORINO & TOTALE \\
\hline 1 & 2 & 9 & 6 & 1 & 19 \\
\hline \multirow[t]{2}{*}{ I } & & & 5 & & 6 \\
\hline & 1 & & 3 & & 4 \\
\hline 2 & & & & & 2 \\
\hline \multirow[t]{3}{*}{3} & & 3 & & & 6 \\
\hline & & 1 & & & 1 \\
\hline & & I & & & 1 \\
\hline
\end{tabular}

Tabella 3. Malattie infettive soggette a notifica accertate con ricerca di antigeni/tossine

\begin{tabular}{|c|c|c|c|c|c|}
\hline \multirow{3}{*}{ REPARTO } & \multicolumn{5}{|c|}{ Ag / TOSSINE A, B } \\
\hline & CLOSTRIDIUM D. & LEGIONELLA P & RSV & ADENOVIRUS & ROTAVIRUS \\
\hline & $\mathrm{FECl}$ & URINE & $\mathrm{TN}$ & $\mathrm{FECl}$ & $\mathrm{FECl}$ \\
\hline CARDIOLOGIA & 5 & & & & \\
\hline CHIRURGIE & 7 & & & & \\
\hline M. INFETTIVE & 21 & 1 & & & \\
\hline MEDICINE & 37 & 8 & & & \\
\hline NEFROLOGIA & 12 & & & & \\
\hline NEUROCHIRURGIA & 1 & & & & \\
\hline ORTOPEDIE & I & & & & \\
\hline PEDIATRIA & 2 & & 13 & 20 & 36 \\
\hline T. INTENSIVE & 4 & 1 & 2 & & \\
\hline TOTALE & 90 & 10 & 15 & 20 & 36 \\
\hline
\end{tabular}


Tabella 4. Casi di TBC e micobatteriosi soggetti a notifica accertati con uno dei tre metodi

\begin{tabular}{|c|c|c|c|c|c|c|c|c|c|c|}
\hline \multirow{3}{*}{ REPARTO } & \multicolumn{9}{|c|}{ METODI/MATERIALI } & \multirow{3}{*}{ TOTALE } \\
\hline & \multicolumn{4}{|c|}{ MICROSCOPICO } & \multicolumn{3}{|c|}{ COLTURALE } & \multicolumn{2}{|c|}{ SONDA GENICA } & \\
\hline & ESP & EP & BIOP & $U$ & ESP & EP & BIOP & BIOP & ESP & \\
\hline CHIRURGIA & 1 & & 1 & & & & 3 & & & \\
\hline M. INFETTIVE & 5 & I & & & 3 & & & I & I & \\
\hline MEDICINA & & & & & I & I & & I & & \\
\hline NEFROLOGIA & & & & I & I & & & & & \\
\hline OTORINO & & & I & & & & & & & \\
\hline TOTALE & 6 & I & 2 & 1 & 5 & I & 3 & 2 & I & 22 \\
\hline
\end{tabular}

MATERIALI: ESP=ESPETTORATO; EP=ESSUDATO PLEURICO; BIOP=MATERIALE BIOPTICO; U=URINE

Tabella 5. Casi di parassitosi intestinali ed ematiche soggette a notifica accertati con EMOSCOPIA/ICT

\begin{tabular}{|c|c|c|c|c|c|}
\hline \multicolumn{6}{|c|}{ REPARTI } \\
\hline PARASSITI INTESTINALI & \multicolumn{2}{|c|}{ PEDIATRIA } & \multicolumn{2}{|c|}{ MAL. INFETTIVE } & TOTALE \\
\hline Giardia intestinalis & & & \multicolumn{2}{|l|}{5} & 5 \\
\hline Enterobius vermicularis & \multicolumn{2}{|c|}{3} & & & 3 \\
\hline Taenia spp & & & \multicolumn{2}{|l|}{1} & I \\
\hline TOTALE & & & & & 9 \\
\hline \multirow[t]{2}{*}{ PARASSITI EMATICI } & \multicolumn{2}{|c|}{ PRONTO SOCCORSO } & \multicolumn{2}{|c|}{ MAL. INFETTIVE } & \\
\hline & EMOSCOPIA & ICT & EMOSCOPIA & ICT & \\
\hline Plasmodium falciparum & & & 2 & 2 & 2 \\
\hline Plasmodium vivax & 1 & I & 2 & 2 & 3 \\
\hline TOTALE & & & & & 5 \\
\hline
\end{tabular}

\section{CONCLUSIONI}

È stato sperimentato nella nostra realtà ospedaliera un modello organizzativo-funzionale di sorveglianza delle malattie infettive soggette a notifica, fondato sull'attivazione dei flussi informativi, a partire dalla raccolta esaustiva delle informazioni clinico-microbiologiche e dell'osservanza scrupolosa della registrazione e comunicazione documentale.

La nostra esperienza di osservatorio epidemiologico è risultata efficace per la verifica è il sollecito ai Reparti della denuncia obbligatoria delle malattie da infezione.

Le segnalazioni per via breve, da parte del Laboratorio di Microbiologia, hanno consentito, alle U.O. coinvolte, di avviare tempestivamente gli interventi più appropriati d'ordine preventivo, atti ad evitare/ridurre la diffusibilità dei microrganismi responsabili, in relazione alla loro biodiversità.

La mancata segnalazione può ripercuotersi negativamente in termini di igiene ospedaliera e anche in termini di economia sanitaria.

La nostra esperienza in merito alla rilevazione delle malattie da infezione basata sulla organizza- zione sistematica dei flussi informativi e della reportistica inerente, porta a ritenerla strumento efficace per la verifica e per il sollecito ai reparti della denuncia obbligatoria delle malattie infettive diffusibili in ambito nosocomiale.

L'organizzazione di un programma di sorveglianza continua - caratterizzato dalla esaustività, periodicità e regolarità delle rilevazioni, dalla disseminazione dei reports aggiornati tra i diversi attori consapevoli dell'importanza dell'operazione - deve fare ricorso a network elettronici con potenziale informativo rilevante per la gestione dell'epidemiologia ospedaliera $(15,17)$.

Un tale modello così strutturato può essere validamente esteso anche alla prevenzione delle: epidemie nosocomiali, infezioni difficili da trattare sostenute da microrganismi problematici, insorgenze delle antibiotico-resistenze (20).

\section{BIBLIOGRAFIA}

1. Aristolao R, Arosio M, Averana F, et al. Sorveglianza delle malattie infettive a partire dalla microbiologia. $3^{\circ}$ Convegno interdisciplinare sulle infezioni ospedaliere. Bergamo 2002. 
2. Azara A, Masia MD, Maida I, Giaconi GL, Campo P, Mura. Analisi dell'attività dei C.I.O. in ambito regionale e proposta di un modello organizzativo- funzionale. $3^{\circ}$ Convegno interdisciplinare sulle Infezioni Ospedaliere. Bergamo 2002.

3. Carrieri MP, Salmaso S, Bella A, et al. Evaluation of the SIMI system, an experimental computerised network for the Surveillance of communicable diseases in Italy. Istituto Superiore di Sanità. Roma 2000; 16 (10): 941-7.

4. Center For Disease Control (C.D.C.). Outline for Surveillance and control of nosocomial infections. U.S. Departement of Health and Human Services, Public Health Service. Atlanta, 1972.

5. Center For Disease Control (C.D.C.) Case definitions for infectious conditions under public health surveillance.MMWR 1997;46 (No.RR-10).

6. David L, Heiman e Rodier GR. Global Surveillance of communicable diseases. Emerging Infections Diseases. WHO Geneva Switzerland 1988; 4 (3).

7. Moioli F, Aristolao R, Averara F, et al. Segnalazione alla ASL di malattia infettiva per via breve dalla microbiologia. $3^{\circ}$ Convegno interdisciplinare sulle Infezioni Ospedaliere. Bergamo 2002.

8. Moro ML. In: Infezioni Ospedaliere: Prevenzione e controllo. Centro Scientifico Editore. Torino 1993; 1734.

9. Murray et al. In: Manual of Clinical Microbiology $7^{\text {th }}$ Edition. American Society for Microbiology 1999; 1-1773.

10. Pagano A, Privitera G, Moro LM et al. In: Le infezioni ospedaliere- Epidemiologia. La sorveglianza basata sui dati di laboratorio. Intramed Communications Milano 1993; 87-104.

11. Pagano A, Privitera G, Moro LM et al. In: Le infezioni ospedaliere-Epidemiologia.

Pianificazione di un sistema di sorveglianza.
Intramed Communications Milano 1993; 73-86.

12. Pagano A, Privitera G, Moro LM et al. In: Le infezioni ospedaliere-Prevenzione. La prevenzione ed il controllo delle Infezioni Ospedaliere. Intramed Communications Milano 1993; 13-53.

13. Panackal AA, et al. Automatic electronic laboratory. Based reporting of notifiable infectious diseases at a large health system. Emerging Infectious Diseases 2002; 8 (7): 685-91.

14. Raffaele B, Martini L, Nurra G, et al. Esperienza di un sistema di allerta per sorveglianza delle infezioni; $3^{\circ}$ Convegno interdisciplinare sulle Infezioni Ospedaliere. Bergamo 2002.

15. Salmaso S, D'Ancona F. Micronet: un progetto di sorveglianza delle malattie infettive basato su una rete di laboratori di microbiologia. Relazione presentata al Convegno su "Sorveglianza delle infezioni: il ruolo della microbiologia clinica e dei sistemi informatici. Ospedale San Carlo Borromeo. Milano 26 Giugno 2003.

16. Simmons M. Complexity theory in the management of communicable diseases. Journal of Hospital Infections 2003; 54 (2): 87-92.

17. Trish MP, Surveillance, reporting and the use of computers. In: Prevention and control of nosocomial infectious Willians \& Wilkins, Ed. Wenzel RP 1997; 127-161

18. Viganò EF. Esperienze innovative nell'applicazione dei sistemi informatici alla sorveglianza delle infezioni. Relazione presentata al Convegno su "Sorveglianza delle infezioni: il ruolo della microbiologia clinica e dei sistemi informatici. Ospedale San Carlo Borromeo. Milano 26 Giugno 2003

19. Wenzel RP. In: Prevention and Control of Nosocomial Infections 3th Editor. Wenzel RP.

20. World Health Organisation, (W.H.O.) Infectious Disease Report, 2000.

\section{Angelo Sala \\ Laboratorio Patologia Clinica II - Microbiologia, Azienda Ospedaliera “A. Manzoni”, 23900 Via dell'Eremo 9/11; 23900 Lecco Tel.: 0341 489618; Fax: 0341489601 e-mail: a.sala@ospedale.lecco.it}

